Published in final edited form as:

Sleep Med Clin. 2008 March ; 3(1): 37-46.

\title{
Polycystic Ovary Syndrome and Obstructive Sleep Apnea
}

\author{
Esra Tasali, MD [Assistant Professor of Medicine] ${ }^{\mathrm{a}}$, Eve Van Cauter, PhD [Professor of \\ Medicine] $^{\mathrm{b}}$, and David A. Ehrmann, MD [Professor of Medicine] ${ }^{\mathrm{C}}$ \\ aSection of Pulmonary and Critical Care Medicine, Department of Medicine, University of Chicago, Chicago, \\ $I L$ \\ bSection of Endocrinology, Department of Medicine, University of Chicago, Chicago, IL \\ cSection of Endocrinology, Department of Medicine, University of Chicago, Chicago, IL
}

\section{Synopsis}

Polycystic ovary syndrome (PCOS), the most common endocrine disorder of pre-menopausal women, is characterized by chronic hyperandrogenism, oligoanovulation, obesity and insulin resistance. Importantly, PCOS women are at increased risk for glucose intolerance, type 2 diabetes and cardiovascular disorders. Recent reports indicate an unexpectedly high prevalence of obstructive sleep apnea (OSA) in PCOS. Alterations in sex steroids (i.e. high androgen and low estrogen levels) and increased visceral adiposity in PCOS could potentially contribute to the increased prevalence of OSA in this disorder. There is some evidence to suggest that there may be strong associations between the presence and severity of OSA and the metabolic disturbances that characterize PCOS. Causal mechanisms in the link between PCOS and OSA remain to be elucidated. Clinicians who manage PCOS patients should be aware of the high prevalence of OSA in these patients and systematically evaluate these women for sleep disturbances.

\section{Keywords}

PCOS; visceral adiposity; obesity; insulin resistance; OSA

Polycystic ovary syndrome (PCOS) affects approximately 5-8\% of women in the United States, making it the most common endocrine disorder of pre-menopausal women [1]. Women with PCOS have a substantial risk for the development of a number of metabolic $[2,3]$ and cardiovascular [4-6] disorders. Specifically, women with PCOS have among the highest reported rates of early-onset impaired glucose tolerance and type 2 diabetes $[7,8]$ as well as a substantial increase in risk for hypertension [9], dyslipidemia, coronary [10] and other vascular disorders $[11,12]$. A recently identified and potentially very important addition to this list of health risks is obstructive sleep apnea (OSA), which is present in PCOS women at rates that are considerably higher than in age and weight-matched reproductively normal women [13]. Current evidence suggests that, in non-PCOS populations, OSA is independently linked to adverse metabolic and cardiovascular outcome [14-16]. The majority of studies that have

\footnotetext{
Corresponding author David A. Ehrmann, M.D., Department of Medicine, MC 1027, University of Chicago, 5841 South Maryland Ave., Chicago, IL 60637, Tel: (773) 702-9653, Fax: (773) 834-0486, dehrmann@uchicago.edu.

Co-author address Eve Van Cauter, Ph.D., Department of Medicine, MC 1027, University of Chicago, 5841 South Maryland Avenue, Chicago, IL 60637, Tel: (773) 702-0169, Fax: (773) 702-7686, evcauter@ medicine.bsd.uchicago.edu

Co-author address Esra Tasali, M.D., Department of Medicine, MC 6026, University of Chicago, 5841 South Maryland Avenue, Chicago, IL 60637, Tel: (773) 702-1497, Fax: (773) 702-7686, etasali@medicine.bsd.uchicago.edu

Publisher's Disclaimer: This is a PDF file of an unedited manuscript that has been accepted for publication. As a service to our customers we are providing this early version of the manuscript. The manuscript will undergo copyediting, typesetting, and review of the resulting proof before it is published in its final citable form. Please note that during the production process errors may be discovered which could affect the content, and all legal disclaimers that apply to the journal pertain.
} 
characterized metabolic and cardiovascular abnormalities in PCOS have, however, not considered the high prevalence of OSA in this syndrome and the possible contribution of OSA to the metabolic and cardiovascular disorders in PCOS.

In the present chapter, we provide a brief introduction to PCOS and review the current notions regarding the pathogenesis of the metabolic and cardiovascular abnormalities in PCOS. Studies that have examined the prevalence of OSA in PCOS and the evidence for a role of sex steroids in promoting OSA in women with PCOS are summarized, and the possible role of OSA in the increased risk of insulin resistance and type 2 diabetes associated with PCOS is discussed.

\section{PCOS: definition and pathogenesis}

A diagnosis of PCOS requires that at least two of the three following abnormalities are present: 1. absent or irregular menstrual cycles; 2 . elevated levels of circulating androgens or clinical manifestations of hyperandrogenism (e.g. hirsutism, acne, alopecia); 3. polycystic ovaries on ultrasonography [17]. Thus, polycystic ovaries need not be present in PCOS women, and conversely, the presence of polycystic ovaries alone does not establish a diagnosis of PCOS. PCOS typically manifests at the time of puberty with menstrual irregularity, hirsutism, and obesity (particularly central adiposity) [17].

\section{Hyperandrogenism}

The cause of hyperandrogenism in PCOS has been elucidated by both in vivo and in vitro studies. In response to stimulation by luteinizing hormone (LH) released by the pituitary gland, the ovarian theca cell synthesizes androstenedione and testosterone. Androstenedione is converted by $17 \beta$-hydroxysteroid-dehydrogenase (17 $\beta$-HSD) to form testosterone or aromatized by the aromatase enzyme (cytochrome P450arom) to form estrone. Theca cells from PCOS ovaries appear to be more efficient at converting androgenic precursors to testosterone than are normal theca cells [18]. Thus, testosterone levels are generally elevated in women with PCOS.

\section{Insulin resistance}

Insulin resistance is frequently present in PCOS women and is considered as a hallmark of the syndrome. In insulin-resistant individuals, higher concentrations of insulin (hyperinsulinemia) are needed to compensate and maintain normal glucose tolerance. It has been well documented that in PCOS, the compensatory hyperinsulinemia contributes both directly and indirectly [19-21] to the increase in plasma androgen concentrations that characterize PCOS. Insulin acts directly by binding to its cognate receptor on the ovarian theca cell to stimulate testosterone synthesis [22]. Insulin can also act indirectly to raise the serum concentration of free testosterone by lowering the serum concentration of sex hormone binding globulin (SHBG) [21].

\section{Progesterone and Estrogen}

Women with PCOS not only have higher levels of androgens but also lower levels of progesterone and estrogen. Over time, circulating progesterone levels in PCOS women are lower than those in normally cycling women $[23,24]$. This appears related to the chronic oligoor anovulation, and the resulting absence or reduction of the normal post-ovulatory surge in progesterone production [25]. Progesterone is thought to "protect" against the development of OSA during pregnancy-associated weight gain [26]. However, as reviewed in Chapter 8 of this issue, there is emerging evidence suggesting the onset, or an increase in severity, of sleepdisordered breathing (especially snoring) in the last trimester of the pregnancy. Physiological and anatomical changes during pregnancy may be conducive to the development of sleepdisordered breathing, particularly in women with preeclampsia (abrupt hypertension, 
hyperalbuminuria, edema in the third trimester). Progesterone is thought to promote its effects through direct stimulation of respiratory drive via an increased ventilatory response to both hypercapnea and hypoxia [27,28]. Progesterone may also act to enhance upper airway dilator muscle activity [29] and to reduce upper airway resistance as has been found post-ovulation in the luteal phase compared to the follicular phase of the menstrual cycle [30]. Reduced progesterone levels in PCOS could be implicated in the high prevalence of OSA.

Underproduction of ovarian estrogen results from low intraovarian aromatase expression and a consequent reduction in the production of the estrogens, estrone and estradiol, from their respective precursor androgens, androstenedione and testosterone. Estrone is also synthesized from peripheral aromatization (especially in adipose tissue) and thus the levels of this steroid are normal or even slightly elevated in PCOS. However, estrone is a weak estrogen with approximately $1 / 10^{\text {th }}$ the potency of estradiol [31]. In sum, estrogen levels are subnormal in PCOS $[32,33]$. It is noteworthy that lower estradiol levels have been reported in association with poor sleep quality among non-PCOS women aged $45-49$ year [34]. Low estradiol levels have also been associated with a higher frequency of apneic events in women across a broad age spectrum of 24 to 72 year [35]. The high prevalence of OSA and the related poor sleep quality in PCOS could be thus also be associated with the low estrogen levels that characterize this condition.

\section{Metabolic and ardiovascular abnormalities in PCOS}

Women with PCOS are more insulin resistant than weight-matched control women and have an increased risk of hypertension, dyslipidemia and cardiovascular disorders [17]. The vast majority of studies that have focused on the metabolic and cardiovascular abnormalities of PCOS have not controlled for the presence of OSA.

There is ample evidence to support a causal link between hyperinsulinemia and the characteristic features of PCOS. For example, reduction of serum insulin levels in women with PCOS results in a decrease in ovarian androgen biosynthesis, an increased SHBG concentration, and a resultant decrease in free testosterone concentrations [2,36]. Insulin also plays a key role in the higher risk of impaired glucose tolerance and type 2 diabetes of PCOS [2,36]. Attenuation of hyperinsulinemia, whether through weight reduction [37] or administration of either metformin [38] or a thiazolidinedione [39-41] substantially attenuates the metabolic perturbations of PCOS.

While obesity is a major factor in the development of insulin resistance in PCOS, it is now established that a component of insulin resistance in PCOS is independent of body weight $[36,42]$. Both lean and obese women with PCOS are more insulin resistant than their non-PCOS counterparts matched for total and fat-free body mass as documented using a variety of wellestablished techniques to assess insulin sensitivity $[36,40,42,43]$. The potential role of OSA in the severity of insulin resistance in PCOS remains to be elucidated. Reduced sleep duration and quality have been shown to result in decreased insulin sensitivity in laboratory studies conducted in healthy young non-obese adults, and the majority of individuals with OSA have reduced sleep duration and quality [44].

In long-term follow-up studies of women with PCOS there is an increased prevalence of type 2 diabetes when compared to appropriate controls [9]. Two large, prospective studies in PCOS place the prevalence of impaired glucose tolerance (IGT) between 30-40\% and type 2 diabetes between 5-10\% [7,8]. These prevalences approach those in Pima Indians, a population with one of the highest rates of development of type 2 diabetes [45]. More recently, we [7] and others [46] have found that the conversion rates from normal glucose tolerance to IGT or type 2 diabetes in PCOS are substantially elevated. 
Insulin secretory defects also play an important role in the propensity to develop diabetes in PCOS. Initial evidence for $\beta$-cell dysfunction in PCOS was derived from analyses of basal and postprandial insulin secretory responses in women with PCOS relative to weight-matched controls with normal androgen levels [47]. The incremental insulin secretory response to meals was markedly reduced in non-diabetic women with PCOS and presented striking similarities with the pattern of meal responses typical of individuals with established type 2 diabetes [48]. Reduced beta-cell responsiveness to glucose may be experimentally produced in normal healthy individuals by recurrent partial sleep deprivation or reduction of sleep quality, suggesting that sleep disturbances in PCOS may contribute to insulin secretory deficits.

Insulin secretion is most appropriately expressed in relation to the magnitude of ambient insulin resistance. When the initial insulin response to intravenous glucose is analyzed in relation to the degree of insulin resistance, women with PCOS exhibit a significant impairment in $\beta$-cell function $[39,43]$. $\beta$-cell function in PCOS was also quantified by examining the insulin secretory response to a graded increase in plasma glucose and the ability of the $\beta$-cell to adjust and respond to induced oscillations in the plasma glucose level [43]. Results from both provocative stimuli were consistent: when expressed in relation to the degree of insulin resistance, insulin secretion was impaired in PCOS subjects.

Women with PCOS are frequently characterized as having elevated triglyceride (TG) levels, increased levels of very low density lipoproteins (VLDL) and LDL, and a lower HDL cholesterol [49], a lipid pattern similar to that seen in patients with type 2 diabetes. The mechanisms responsible for the adverse effects of PCOS on plasma TG homeostasis are not known. Insulin resistance has been postulated to play a key role in causing hypertriglyceridemia in PCOS. However, we found that treatment with the insulin sensitizing agent troglitazone markedly improved insulin sensitivity in PCOS women but had little, if any, effect on plasma TG concentration [50]. In addition, lean women with PCOS are found to have normal plasma TG concentrations despite being hyperinsulinemic [10].

Both lipid and non-lipid criteria identify individuals at increased risk for coronary heart disease and type 2 diabetes [51,52]. Because women with PCOS have high rates of impaired glucose tolerance and type 2 diabetes [7,8] as well as a substantial number of risk factors for cardiovascular disease [50], it has been generally assumed that many are also at increased cardiometabolic risk.

\section{Prevalence of OSA in PCOS}

The most recent estimate of the prevalence of OSA in the general adult population is approximately $17 \%$. In overweight individuals (body mass index, BMI $\geq 25 \mathrm{~kg} / \mathrm{m}^{2}$ ), the proportion of mild to moderate OSA is 41 to 58\% [53]. As discussed in Chapters 8 and 12 of this issue of Sleep Medicine Clinics, both community and clinic-based studies have consistently noted that men have a higher prevalence of OSA than women [54-57]. The prevalence of OSA is particularly low in pre-menopausal women and increases after menopause $[54,58,59]$ (see Chapter 11 of this issue). Among women with PCOS, however, OSA is considerably more common than expected [13,60,61]. Vgontzas et al. assessed the prevalence of OSA in 53 premenopausal women with PCOS as compared to 452 control women [60]. The authors found that PCOS women were 30 times more likely to have OSA than controls and that the difference between the two groups remained significant even after controlling for BMI. In an independent study published the same year, comparison of 18 overweight women with PCOS with 18 ageand weight-matched controls, showed that PCOS women were significantly more likely to suffer from symptomatic OSA (based on an apnea hypopnea index (AHI) $>5$ and the presence of excessive daytime sleepiness) than control women (44.4\% vs 5.5\%) [13]. In another cohort of 23 obese PCOS women, the prevalence of OSA was found to be $70 \%$ and there was no 
association between obesity (as assessed by BMI) and the severity of OSA [61]. Recently, survey assessments of the prevalence of sleep apnea risk (using the Berlin questionnaire) in a cohort of 40 women with PCOS, revealed that three of four women were at high risk for sleep apnea [62]. About two third of these PCOS women had poor sleep quality as assessed by the Pittsburgh Sleep Quality questionnaire and $45 \%$ had chronic daytime sleepiness as defined by the Epworth Sleepiness Scale. Remarkably, less than $8 \%$ of this cohort of 40 women with PCOS were free of sleep complaints (Figure 1) [62]. Consistent with the findings of frequent daytime somnolence, in two other studies, women with PCOS were found to be subjectively sleepier (as defined by the Epworth Sleepiness Scale) [13] and nine times more likely (80.4\% vs $27.0 \%$ ) to report daytime sleepiness [60] than controls. Sleep disturbances thus appear to be an important feature of PCOS. Given the high prevalence of OSA among women with PCOS, it may be warranted to systematically evaluate them for sleep disorders. However, findings from a recent survey suggest that more than $90 \%$ of physicians who manage PCOS patients rarely ordered a sleep study (less than $25 \%$ of the time) [63].

\section{PCOS and OSA: role of sex steroids and obesity}

\section{Sex steriods}

Differences in concentrations of circulating sex steroids i.e. estrogens, progestins, and androgens are believed to partly account for the gender disparity in normal sleep architecture [64] as well as in the prevalence and severity of OSA [65]. While androgens are thought to be "harmful", estrogens and progestins have been generally characterized as "protective" against the development of OSA. Several studies have shown that testosterone administration may have adverse effects on breathing during sleep and predispose to OSA [65-67]. Testosterone appears to influence both the neural control of breathing [68] and upper airway mechanics [69]. Zhou et al. [67] examined the effect of testosterone on the hypocapnic apneic threshold in women during sleep. Eight normal, healthy, pre-menopausal women were studied before and after treatment with transdermal testostosterone $(5 \mathrm{mg} /$ day) administered in the follicular phase of the menstrual cycle. The authors concluded that testosterone increases the apneic threshold in premenopausal women, facilitating central apneas, thus leading to breathing instability during sleep.

Although it is plausible that androgen excess, a defining feature of PCOS, contributes to the higher prevalence of OSA in this disorder, it appears that factors other than the androgen excess may be involved in the increased prevalence of OSA in PCOS. Indeed, two studies found no significant correlations between androgen levels and the severity of OSA [60,62]. In one study of women with PCOS $(n=53)$, those taking oral contraceptives $(n=14)$ were less likely to have OSA [60], consistent with the concept of a "protective role" for estrogens and progesterone in the pathogenesis of OSA [54,58,59]. In contrast, Fogel et al. reported that the severity of OSA (as assessed by the apnea-hypopnea index) correlated with both total and free testosterone levels [13].

\section{Obesity}

The link between obesity and OSA has long been recognized [53]. However, it seems that high prevalence of OSA in PCOS is not simply due to the elevated BMI $[13,60,61]$ but may be more closely related to the degree of visceral adiposity. Visceral fat appears to be more metabolically active and the quantity of visceral fat has been shown to highly correlate with OSA risk [53]. The relative proportion of visceral fat to total body fat is higher in obese men compared to obese women. This difference is thought to contribute to the higher prevalence of OSA in men than women. Women with PCOS have typically a high prevalence of visceral adiposity, as assessed, for example, by higher waist to hip circumferences than non-PCOS BMI-matched women. Fogel et al. reported a significant relationship between the waist to hip ratio, a measure 
of central obesity, and the severity of OSA in women with PCOS [13]. The authors suggested that high androgen levels promote central obesity, which in turn leads to OSA. The latter finding is consistent with the studies in non-PCOS populations indicating that visceral obesity is closely associated with OSA [53].

In summary, the current evidence suggests that the increased prevalence of OSA in PCOS cannot be fully explained by elevated BMI and high androgen levels. Further well-controlled studies with careful characterization of the levels of sex steroids as well as assessments of body fat distribution are needed to fully elucidate the complex interactions between obesity, androgen excess and the presence and severity of OSA in the PCOS population.

\section{PCOS and OSA: insulin resistance, glucose intolerance and type 2 diabetes}

In non-PCOS populations, there is substantial evidence from cross-sectional studies that supports an independent association between the presence and severity of OSA, and alterations in glucose metabolism including glucose intolerance, insulin resistance and type 2 diabetes [14-16]. A number of interventional studies have shown that the treatment of OSA with continuous airway positive pressure (CPAP) improves insulin sensitivity [70] and is associated with a reduction in postprandial glucose and glycohemoglobin levels in individuals with type 2 diabetes [71]. So far, only two previous studies have examined the relationships between OSA and alterations in glucose metabolism in women with PCOS [60,62].

In the first study, Vgontzas and colleagues [60] found that PCOS patients who were recommended treatment for OSA (based on the severity of the symptoms) had significantly higher plasma insulin levels and a lower glucose-to-insulin ratio than those who did not have clinically significant OSA, independently of BMI. These findings suggest that in the presence of clinically significant OSA, women with PCOS may present with a more severe insulin resistant state. Using a logistic regression model, the authors also concluded that insulin resistance was the strongest predictor of OSA after controlling for age, BMI, and circulating testosterone concentrations [60].

In the second study, we [62] explored the relationships between glucose metabolism and OSA in two cohorts of non-diabetic PCOS women. In the first cohort, sleep apnea risk was determined by the Berlin questionnaire and glucose metabolism was assessed by oral glucose tolerance test (OGTT) in 32 women with PCOS. Fasting insulin levels and homeostatic model assessment (HOMA; defined as the normalized product of fasting glucose by fasting insulin and used as a measure of insulin resistance) index were significantly higher in the women who were at high risk for sleep apnea. Furthermore, among the 19 women with normal glucose tolerance, the insulin response to glucose ingestion was more than two-fold higher in women at high risk for sleep apnea as compared to those at low risk (Figure 2). The latter findings suggest that sleep apnea may contribute to the accelerated conversion rate from normal to impaired glucose tolerance in PCOS.

In the second cohort [62], we assessed eight women with PCOS by overnight polysomnography and OGTT. The women with PCOS had reduced sleep efficiency compared to age-matched, healthy non-obese controls (mean \pm SEM: $80 \pm 5 \%$ versus $92 \pm 1 \%$ ), and reduced REM sleep time - at about half that of the control group (mean \pm SEM: $46 \pm 10$ versus $96 \pm 8$ minutes). Women with OSA tend to have a clustering of events during REM sleep [72], and our cohort of the women with PCOS were found to have severe REM-related OSA with a mean \pm SEM AHI of $42 \pm 6$ per hour of REM sleep. In this small cohort of women with PCOS, remarkable associations between measures of glucose tolerance and the severity of OSA were detected. Specifically, the markers of the severity of OSA, as quantified by the total AHI of $7 \pm 1$ per hour of sleep (mean \pm SEM) or the number of desaturations, by at least $3 \%$ from baseline, in 
REM sleep (the mean \pm SEM of the oxygen desaturation index was $36 \pm 4$ per hour of REM sleep), were strong predictors of glucose tolerance (Figure 3).

Several studies have demonstrated that insulin resistance, visceral fat and higher circulating levels of pro-inflammatory cytokines are independently and strongly associated with OSA and excessive daytime sleepiness [73,74]. In a recent report [75] comparing 42 obese PCOS women without OSA, 17 obese women without OSA and 15 normal weight controls, it was shown that interleukin-6 levels were elevated in obese women with PCOS independently of obesity or OSA, suggesting that pro-inflammatory cytokines may be one of the pathways leading to insulin resistance in PCOS.

In summary, limited evidence suggests the existence of strong associations between OSA and abnormalities in glucose metabolism among women with PCOS. Causal mechanisms underlying these relationships remain to be elucidated. In particular, systematic screening for OSA and carefully designed interventional studies to examine the impact of CPAP treatment of OSA would be important in women with PCOS and may lead to better treatment options.

\section{Summary}

PCOS is one of the most common endocrine disorders affecting women. This syndrome involves a number of reproductive, metabolic and cardiovascular abnormalities. Importantly, affected women are at increased risk for the development of early-onset impaired glucose tolerance and type 2 diabetes. Androgen excess and insulin resistance are the main characteristics in the pathogenesis of PCOS. Recent evidence is that sleep disturbances also appear to be an important feature in PCOS and that OSA is considerably more common than expected in women with PCOS. However, the high prevalence of OSA is under recognized among clinicians who manage PCOS patients. Limited evidence summarized in this review suggests that the androgen excess, subnormal estrogen levels and visceral adiposity could be involved in the increased risk of OSA in PCOS, and that there may be a strong association between the severity of OSA and glucose intolerance and insulin resistance. Further research is needed to fully elucidate the causal mechanisms. A better understanding of the link between OSA and PCOS could lead to better treatment options. Systematic screening for OSA in women with PCOS may be warranted.

\section{Acknowledgements}

This work was supported by RO1 HL075079 and P50 HD057796 to D.A.E, Scholars Grants in Sleep Medicine to E.T. and PO1 AG-11412 to E.V.C.

\section{References}

1. Knochenhauer E, Key T, Kahsar-Miller M, et al. Prevalence of polycystic ovary syndrome inunselected black and white women of the southeastern United States: a prospective study. J Clin Endocrinol Metab 1998;83:3078-3082. [PubMed: 9745406]

2. Ehrmann DA, Barnes RB, Rosenfield RL. Polycystic ovary syndrome as a form of functional ovarian hyperandrogenism due to dysregulation of androgen secretion. Endocr Rev 1995;16(3):322-353. [PubMed: 7671850]

3. Dunaif A, Segal KR, Futterweit W, et al. Profound peripheral insulin resistance, independent of obesity, in polycystic ovary syndrome. Diabetes 1989;38(9):1165-1174. [PubMed: 2670645]

4. Talbott E, Guzick D, Clerici A, et al. Coronary heart disease risk factors in women with polycystic ovary syndrome. Arterioscler Thromb Vasc Biol 1995;15(7):821-826. [PubMed: 7600112]

5. Talbott E, Clerici A, Berga SL, et al. Adverse lipid and coronary heart disease risk profiles in young women with polycystic ovary syndrome: results of a case-control study. J Clin Epidemiol 1998;51(5): 415-422. [PubMed: 9619969] 
6. Talbott EO, Guzick DS, Sutton-Tyrrell K, et al. Evidence for association between polycystic ovary syndrome and premature carotid atherosclerosis in middle-aged women. Arterioscler Thromb Vasc Biol 2000;20(11):2414-2421. [PubMed: 11073846]

7. Ehrmann DA, Barnes RB, Rosenfield RL, et al. Prevalence of impaired glucose tolerance and diabetes in women with polycystic ovary syndrome. Diabetes Care 1999;22(1):141-146. [PubMed: 10333916]

8. Legro RS, Kunselman AR, Dodson WC, et al. Prevalence and predictors of risk for type 2 diaetes mellitus and impaired glucose tolerance in polycystic ovary syndrome: a prospective, controlled study in 254 affected women. J Clin Endocrinol Metab 1999;84(1):165-169. [PubMed: 9920077]

9. Dahlgren E, Johansson S, Lindstedt G, et al. Women with polycystic ovary syndrome wedge resected in 1956 to 1965: a long-term follow-up focusing on natural history and circulating hormones. Fertil Steril 1992;57(3):505-513. [PubMed: 1740195]

10. Conway GS, Agrawal R, Betteridge DJ, et al. Risk factors for coronary artery disease in lean and obese women with the polycystic ovary syndrome. Clin Endocrinol (Oxf) 1992;37(2):119-125. [PubMed: 1395062]

11. Kelly CJ, Speirs A, Gould GW, et al. Altered vascular function in young women with polycystic ovary syndrome. J Clin Endocrinol Metab 2002;87(2):742-746. [PubMed: 11836314]

12. Paradisi G, Steinberg HO, Shepard MK, et al. Troglitazone therapy improves endothelial function to near normal levels in women with polycystic ovary syndrome. J Clin Endocrinol Metab 2003;88(2): 576-580. [PubMed: 12574183]

13. Fogel RB, Malhotra A, Pillar G, et al. Increased prevalence of obstructive sleep apnea syndrome in obese women with polycystic ovary syndrome. J Clin Endocrinol Metab 2001;86(3):1175-1180. [PubMed: 11238505]

14. Punjabi NM, Polotsky VY. Disorders of glucose metabolism in sleep apnea. Journal of Applied Physiology 2005;99:1998-2007. [PubMed: 16227461]

15. Wolk R, Somers VK. Sleep and the metabolic syndrome. Exp Physiol 2007;92(1):67-78. [PubMed: 17085678]

16. Tasali, E.; Mokhlesi, B.; Van Cauter, E. Chest. 2008. Obstructive Sleep Apnea and Type 2 Diabetes: Interacting Epidemics. (in press)

17. Ehrmann DA. Polycystic ovary syndrome. N Engl J Med 2005;352(12):1223-1236. [PubMed: 15788499]

18. Nelson VL, Qin KN, Rosenfield RL, et al. The biochemical basis for increased testosterone production in theca cells propagated from patients with polycystic ovary syndrome. J Clin Endocrinol Metab 2001;86(12):5925-5933. [PubMed: 11739466]

19. Barbieri RL, Makris A, Randall RW, et al. Insulin stimulates androgen accumulation in incubations of ovarian stroma obtained from women with hyperandrogenism. J Clin Endocrinol Metab 1986;62 (5):904-910. [PubMed: 3514651]

20. Botwood N, Hamilton-Fairley D, Kiddy D, et al. Sex hormone-binding globulin and female reproductive function. J Steroid Biochem Mol Biol 1995;53(1-6):529-531. [PubMed: 7626505]

21. Buyalos RP, Geffner ME, Watanabe RM, et al. The influence of luteinizing hormone and insulin on sex steroids and sex hormone-binding globulin in the polycystic ovarian syndrome. Fertil Steril 1993;60(4):626-633. [PubMed: 8405515]

22. Nestler JE, Jakubowicz DJ, de Vargas AF, et al. Insulin stimulates testosterone biosynthesis by human thecal cells from women with polycystic ovary syndrome by activating its own receptor and using inositolglycan mediators as the signal transduction system. J Clin Endocrinol Metab 1998;83(6): 2001-2005. [PubMed: 9626131]

23. Fleming R, McQueen D, Yates RW, et al. Spontaneous follicular and luteal function in infertile women with oligomenorrhoea: role of luteinizing hormone. Clin Endocrinol (Oxf) 1995;43(6):735-739. [PubMed: 8736277]

24. Joseph-Horne R, Mason H, Batty S, et al. Luteal phase progesterone excretion in ovulatory women with polycystic ovaries. Hum Reprod 2002;17(6):1459-1463. [PubMed: 12042261]

25. Blank SK, McCartney CR, Marshall JC. The origins and sequelae of abnormal neuroendocrine function in polycystic ovary syndrome. Hum Reprod Update 2006;12(4):351-361. [PubMed: 16670102] 
26. Maasilta $\mathrm{P}$, Bachour A, Teramo K, et al. Sleep-related disordered breathing during pregnancy in obese women. Chest 2001;120(5):1448-1454. [PubMed: 11713118]

27. Pien GW, Schwab RJ. Sleep disorders during pregnancy. Sleep 2004;27(7):1405-1417. [PubMed: 15586794]

28. Regensteiner JG, Woodard WD, Hagerman DD, et al. Combined effects of female hormones and metabolic rate on ventilatory drives in women. J Appl Physiol 1989;66(2):808-813. [PubMed: 2540141]

29. Popovic RM, White DP. Upper airway muscle activity in normal women: influence of hormonal status. J Appl Physiol 1998;84(3):1055-1062. [PubMed: 9480969]

30. Driver HS, McLean H, Kumar DV, et al. The influence of the menstrual cycle on upper airway resistance and breathing during sleep. Sleep 2005;28(4):449-456. [PubMed: 16171289]

31. Gutendorf B, Westendorf J. Comparison of an array of in vitro assays for the assessment of the estrogenic potential of natural and synthetic estrogens, phytoestrogens and xenoestrogens. Toxicology 2001;166(1-2):79-89. [PubMed: 11518614]

32. Waldstreicher J, Santoro NF, Hall JE, et al. Hyperfunction of the hypothalamic-pituitary axis in women with polycystic ovarian disease: indirect evidence for partial gonadotroph desensitization. $\mathrm{J}$ Clin Endocrinol Metab 1988;66(1):165-172. [PubMed: 2961784]

33. Strauss, JF, 3rd. The Synthesis and Metabolism of Steroid Hormones. Elsevier Saunders; Philadelphia, PA: 2004. Chapter 4.

34. Hollander LE, Freeman EW, Sammel MD, et al. Sleep quality, estradiol levels, and behavioral factors in late reproductive age women. Obstet Gynecol 2001;98(3):391-397. [PubMed: 11530118]

35. Netzer NC, Eliasson AH, Strohl KP. Women with sleep apnea have lower levels of sex hormones. Sleep Breath 2003;7(1):25-29. [PubMed: 12712394]

36. Dunaif A. Insulin resistance and the polycystic ovary syndrome: mechanism and implications for pathogenesis. Endocr Rev 1997;18(6):774-800. [PubMed: 9408743]

37. Moran LJ, Noakes M, Clifton PM, et al. Dietary composition in restoring reproductive and metabolic physiology in overweight women with polycystic ovary syndrome. J Clin Endocrinol Metab 2003;88 (2):812-819. [PubMed: 12574218]

38. Nestler JE. Metformin and the polycystic ovary syndrome. J Clin Endocrinol Metab 2001;86(3):1430. [PubMed: 11238551]

39. Dunaif A, Scott D, Finegood D, et al. The insulin-sensitizing agent troglitazone improves metabolic and reproductive abnormalities in the polycystic ovary syndrome. J Clin Endocrinol Metab 1996;81 (9):3299-3306. [PubMed: 8784087]

40. Ehrmann DA, Schneider DJ, Sobel BE, et al. Troglitazone improves defects in insulin action, insulin secretion, ovarian steroidogenesis, and fibrinolysis in women with polycystic ovary syndrome. J Clin Endocrinol Metab 1997;82(7):2108-2116. [PubMed: 9215280]

41. Azziz R, Ehrmann D, Legro RS, et al. Troglitazone improves ovulation and hirsutism in the polycystic ovary syndrome: a multicenter, double blind, placebo-controlled trial. J Clin Endocrinol Metab 2001;86(4):1626-1632. [PubMed: 11297595]

42. Dunaif A, Graf M, Mandeli J, et al. Characterization of groups of hyperandrogenic women with acanthosis nigricans, impaired glucose tolerance, and/or hyperinsulinemia. J Clin Endocrinol Metab 1987;65(3):499-507. [PubMed: 3305551]

43. Ehrmann DA, Sturis J, Byrne MM, et al. Insulin secretory defects in Polycystic Ovary Syndrome: Relationship to insulin sensitivity and family history of non-insulin-dependent diabetes mellitus. J Clin Invest 1995;96:520-527. [PubMed: 7615824]

44. Spiegel K, Knutson K, Leproult R, et al. Sleep loss: a novel risk factor for insulin resistanceand Type 2 diabetes. J Appl Physiol 2005;99(5):2008-2019. [PubMed: 16227462]

45. Gabir MM, Hanson RL, Dabelea D, et al. The 1997 American Diabetes Association and 1999 World Health Organization criteria for hyperglycemia in the diagnosis and prediction of diabetes. Diabetes Care 2000;23(8):1108-1112. [PubMed: 10937506]

46. Legro RS, Gnatuk CL, Kunselman AR, et al. Changes in Glucose Tolerance Over Time in Women with Polycystic Ovary Syndrome: A Controlled Study. J Clin Endocrinol Metab 2005;90(6):32363242. [PubMed: 15797965] 
47. O'Meara NM, Blackman JD, Ehrmann DA, et al. Defects in beta-cell function in functional ovarian hyperandrogenism. J Clin Endocrinol Metab 1993;76(5):1241-1247. [PubMed: 8496316]

48. Polonsky KS, Given BD, Hirsch LJ, et al. Abnormal patterns of insulin secretion in noninsulindependent diabetes mellitus. N Engl J Med 1988;318:1231-1239. [PubMed: 3283554]

49. Pirwany IR, Fleming R, Greer IA, et al. Lipids and lipoprotein subfractions in women with PCOS: relationship to metabolic and endocrine parameters. Clin Endocrinol (Oxf) 2001;54(4):447-453. [PubMed: 11318779]

50. Legro RS, Azziz R, Ehrmann D, et al. Minimal response of circulating lipids in women with polycystic ovary syndrome to improvement in insulin sensitivity with troglitazone. J Clin Endocrinol Metab 2003;88(11):5137-5144. [PubMed: 14602740]

51. Third Report of the National Cholesterol Education Program (NCEP). Expert Panel on Detection, Evaluation, and Treatment of High Blood Cholesterol in Adults (Adult Treatment Panel III) final report. Circulation 2002;106(25):3143-3421. [PubMed: 12485966]

52. Ford ES. The metabolic syndrome and mortality from cardiovascular disease and all-causes: findings from the National Health and Nutrition Examination Survey II Mortality Study. Atherosclerosis 2004;173(2):309-314. [PubMed: 15064107]

53. Young T, Peppard PE, Taheri S. Excess weight and sleep-disordered breathing. J Appl Physiol 2005;99(4):1592-1599. [PubMed: 16160020]

54. Bixler EO, Vgontzas AN, Lin HM, et al. Prevalence of sleep-disordered breathing in women: effects of gender. Am J Respir Crit Care Med 2001;163(3 Pt 1):608-613. [PubMed: 11254512]

55. Young T, Palta M, Dempsey J, et al. The occurrence of sleep-disordered breathing among middleaged adults. N Engl J Med 1993;328(17):1230-1235. [PubMed: 8464434]

56. Young T, Peppard PE, Gottlieb DJ. Epidemiology of obstructive sleep apnea: a population health perspective. Am J Respir Crit Care Med 2002;165(9):1217-1239. [PubMed: 11991871]

57. Young T, Skatrud J, Peppard PE. Risk factors for obstructive sleep apnea in adults. Jama 2004;291 (16):2013-2016. [PubMed: 15113821]

58. Shahar E, Redline S, Young T, et al. Hormone replacement therapy and sleep-disordered breathing. Am J Respir Crit Care Med 2003;167(9):1186-1192. [PubMed: 12531779]

59. Young T, Finn L, Austin D, et al. Menopausal status and sleep-disordered breathing in the Wisconsin Sleep Cohort Study. Am J Respir Crit Care Med 2003;167(9):1181-1185. [PubMed: 12615621]

60. Vgontzas AN, Legro RS, Bixler EO, et al. Polycystic ovary syndrome is associated with obstructive sleep apnea and daytime sleepiness: role of insulin resistance. J Clin Endocrinol Metab 2001;86(2): 517-520. [PubMed: 11158002]

61. Gopal M, Duntley S, Uhles M, et al. The role of obesity in the increased prevalence of obstructive sleep apnea syndrome in patients with polycystic ovarian syndrome. Sleep Med 2002;3(5):401-404. [PubMed: 14592171]

62. Tasali E, Van Cauter E, Ehrmann DA. Relationships between sleep disordered breathing and glucose metabolism in polycystic ovary syndrome. J Clin Endocrinol Metab 2006;91(1):36-42. [PubMed: 16219719]

63. Subramanian S, Desai A, Joshipura M, et al. Practice patterns of screening for sleep apnea in physicians treating PCOS patients. Sleep Breath 2007;11(4):233-237. [PubMed: 17541663]

64. Driver HS, Dijk DJ, Werth E, et al. Sleep and the sleep electroencephalogram across the menstrual cycle in young healthy women. J Clin Endocrinol Metab 1996;81(2):728-735. [PubMed: 8636295]

65. Liu K, Yee B, Philips C, et al. Sleep Apnea and Neuroendocrine Function. Sleep Medicin Clinics 2007;2:225-236.

66. Liu PY, Yee B, Wishart SM, et al. The short-term effects of high-dose testosterone on sleep, breathing, and function in older men. J Clin Endocrinol Metab 2003;88(8):3605-3613. [PubMed: 12915643]

67. Zhou XS, Rowley JA, Demirovic F, et al. Effect of testosterone on the apneic threshold in women during NREM sleep. J Appl Physiol 2003;94:101-107. [PubMed: 12391093]

68. White DP, Schneider BK, Santen RJ, et al. Influence of testosterone on ventilation and chemosensitivity in male subjects. J Appl Physiol 1985;59(5):1452-1457. [PubMed: 4066575] 
69. Cistulli PA, Grunstein RR, Sullivan CE. Effect of testosterone administration on upper airway collapsibility during sleep. Am J Respir Crit Care Med 1994;149(2 Pt 1):530-532. [PubMed: 8306057]

70. Harsch IA, Hahn EG, Konturek PC. Insulin resistance and other metabolic aspects of the obstructive sleep apnea syndrome. Med Sci Monit 2005;11(3):RA70-RA75. [PubMed: 15735578]

71. Babu AR, Herdegen J, Fogelfeld L, et al. Type 2 diabetes, glycemic control, and continuous positive airway pressure in obstructive sleep apnea. Arch Intern Med 2005;165(4):447-452. [PubMed: 15738376]

72. O'Connor C, Thornley KS, Hanly PJ. Gender differences in the polysomnographic features of obstructive sleep apnea. Am J Respir Crit Care Med 2000;161(5):1465-1472. [PubMed: 10806140]

73. Vgontzas AN, Papanicolaou DA, Bixler EO, et al. Sleep apnea and daytime sleepiness and fatigue: relation to visceral obesity, insulin resistance and hypercytokinemia. J Clin Endocrinol Metab 2000;85:1151-1158. [PubMed: 10720054]

74. Vgontzas AN, Papanicolaou DA, Bixler EO, et al. Elevation of Plasma Cytokines in Disorders of Excessive Daytime Sleepiness: Role of Sleep Disturbance and Obesity. Journal of Clinical Endocrinology and Metabolism 1997;82(5):1313-1316. [PubMed: 9141509]

75. Vgontzas AN, Trakada G, Bixler EO, et al. Plasma interleukin 6 levels are elevated in polycystic ovary syndrome independently of obesity or sleep apnea. Metabolism 2006;55(8):1076-1082. [PubMed: 16839844] 


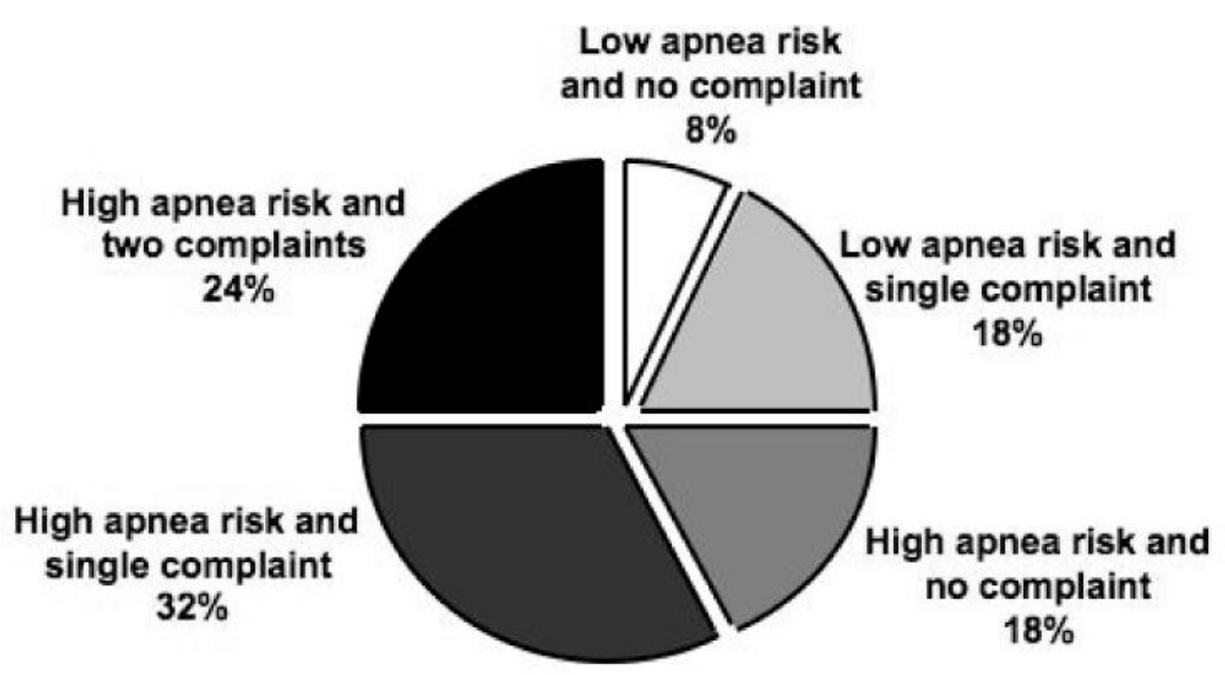

Figure 1.

The frequency distribution of the risk for sleep apnea (derived from responses to the Berlin questionnaire) and sleep complaints derived from responses to the Pittsburgh Sleep Quality (PSQ) questionnaire and Epworth Sleepiness Scale (ESS) in 40 women with PCOS. A single sleep complaint was defined as a PSQ index $>5$ or an ESS score of $\geq 10$. (From Tasali E, Van Cauter E, Ehrmann D. Relationships between sleep disordered breathing and glucose metabolism in the polycystic ovary syndrome. J Clin Endocrinol Metab, 2006, 91:36-42) 
A.

Normal glucose tolerance
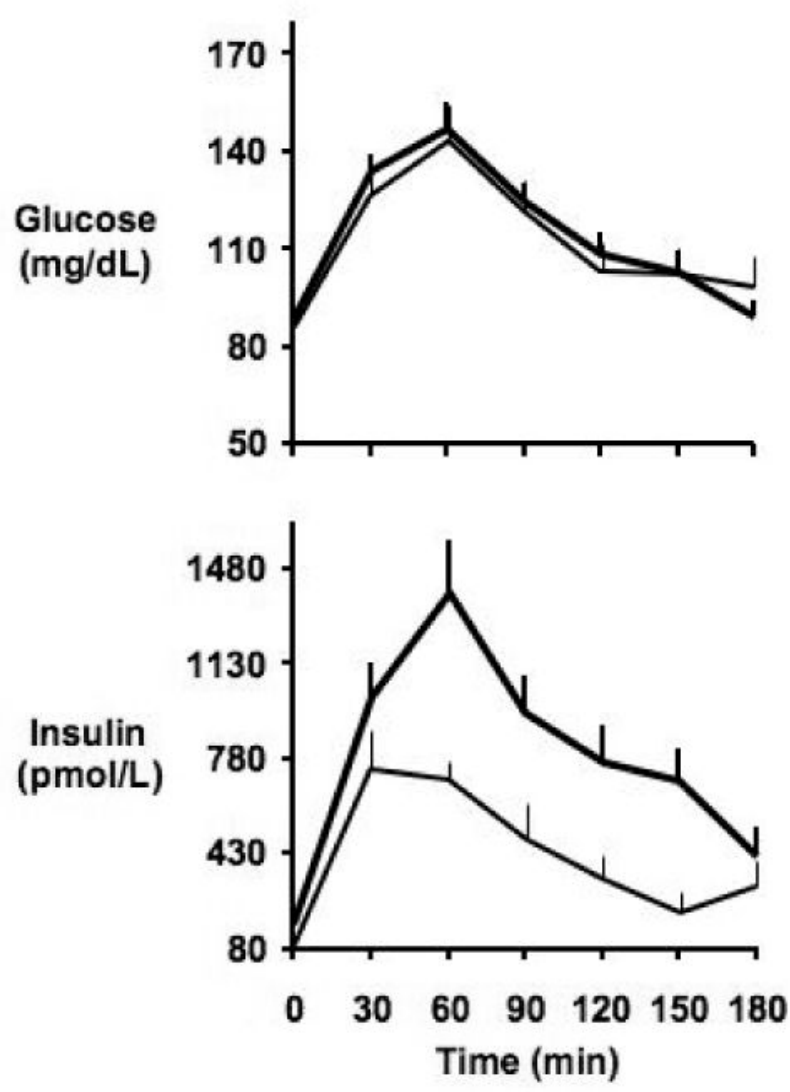

B.

Impaired glucose tolerance
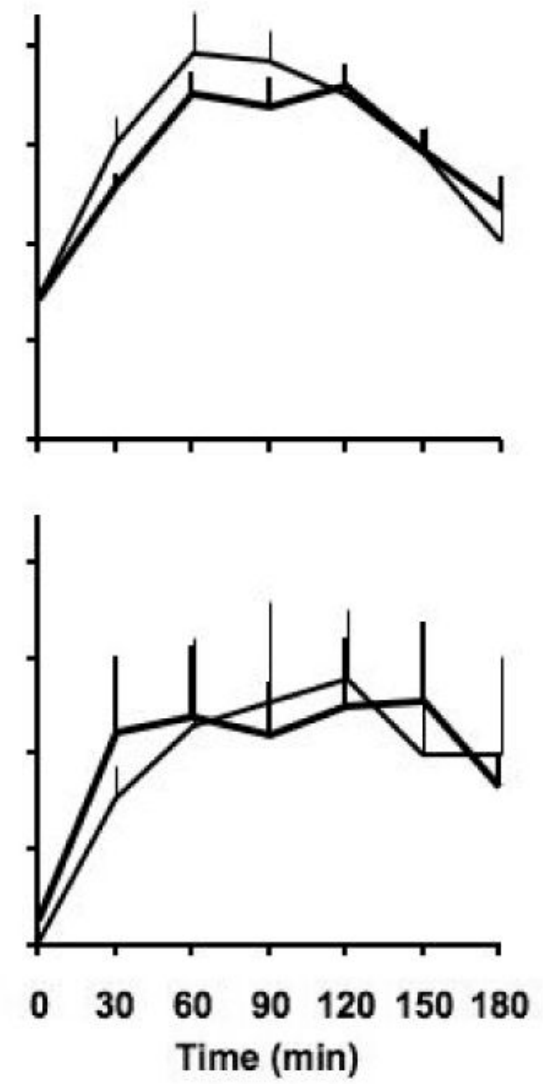

Figure 2.

Mean $( \pm$ SEM) profiles of glucose (upper panel) and insulin (lower panel) concentrations during the oral glucose tolerance test in: A. Women with PCOS with normal glucose tolerance $(n=19)$. B. Women with PCOS with impaired glucose tolerance $(n=13)$. Wide lines represent PCOS women at high risk for sleep apnea; narrow lines represent PCOS women at low risk for sleep apnea. (From Tasali E, Van Cauter E, Ehrmann D. Relationships between sleep disordered breathing and glucose metabolism in the polycystic ovary syndrome. J Clin Endocrinol Metab, 2006, 91:36-42) 

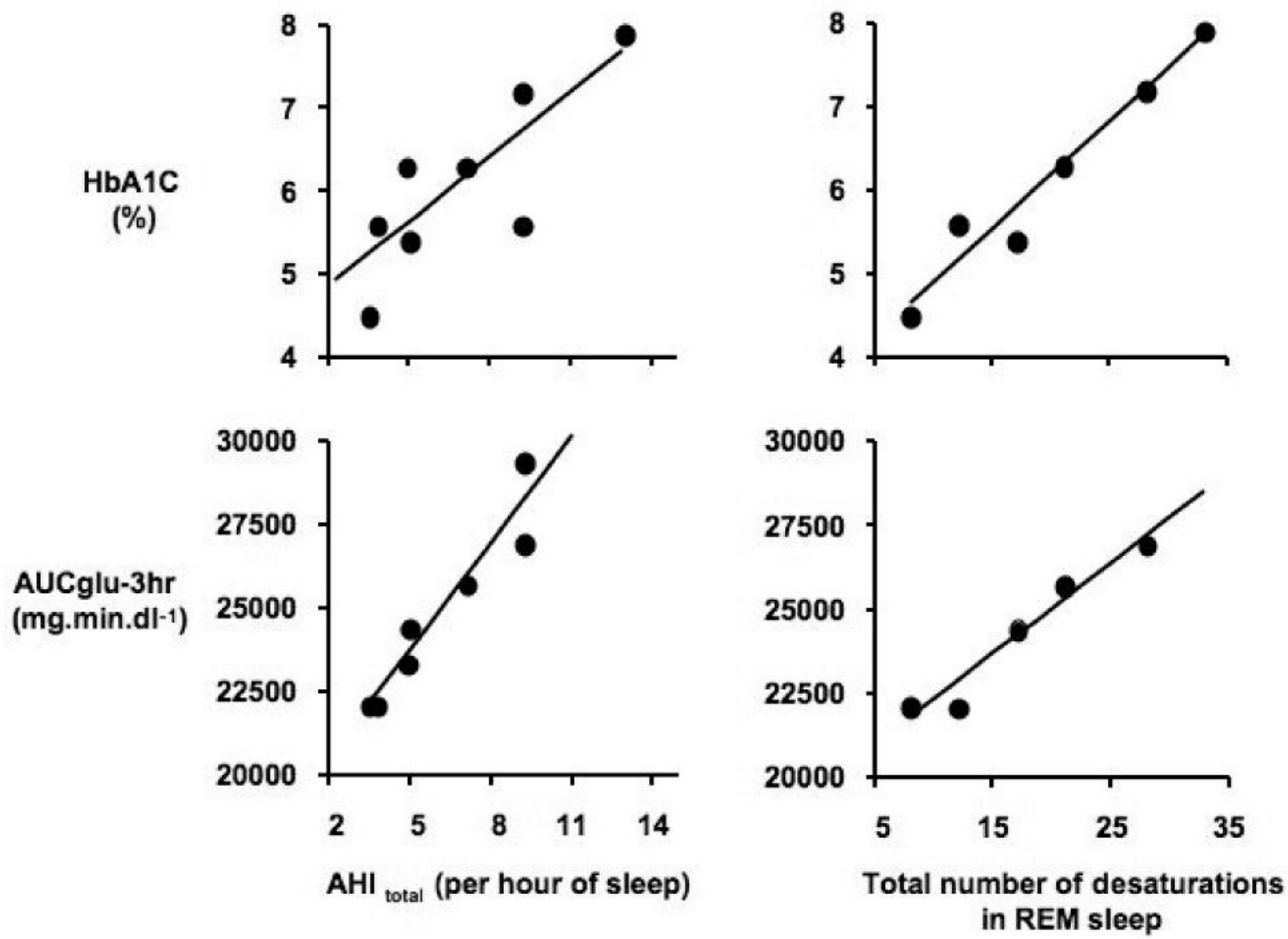

Figure 3.

Relationships between the markers of severity of sleep-disordered breathing (SDB) and measures of glucose tolerance in eight women with PCOS. Left upper panel: relationship between total apnea-hypopnea index ( $\mathrm{AHI}_{\text {total }}$ ) and glycosylated hemoglobin (HbA1C). Left lower panel: relationship between $\mathrm{AHI}_{\text {total }}$ and the area under the glucose curve (AUCglu-3hr). Right upper panel: relationship between total number of $\geq 3 \%$ desaturations in REM sleep and HbA1C. Right lower panel: relationship between total number of $\geq 3 \%$ desaturations in REM sleep and AUCglu-3hr. (From Tasali E, Van Cauter E, Ehrmann D. Relationships between sleep disordered breathing and glucose metabolism in the polycystic ovary syndrome. J Clin Endocrinol Metab, 2006, 91:36-42) 